\title{
Neurobrucellosis: report of a rare disease in 20 Iranian patients referred to a tertiary hospital
}

M. Ranjbar, ${ }^{1}$ A.A. Rezaiee, ${ }^{2}$ S.H. Hashemi ${ }^{1}$ and S. Mehdipour ${ }^{3}$

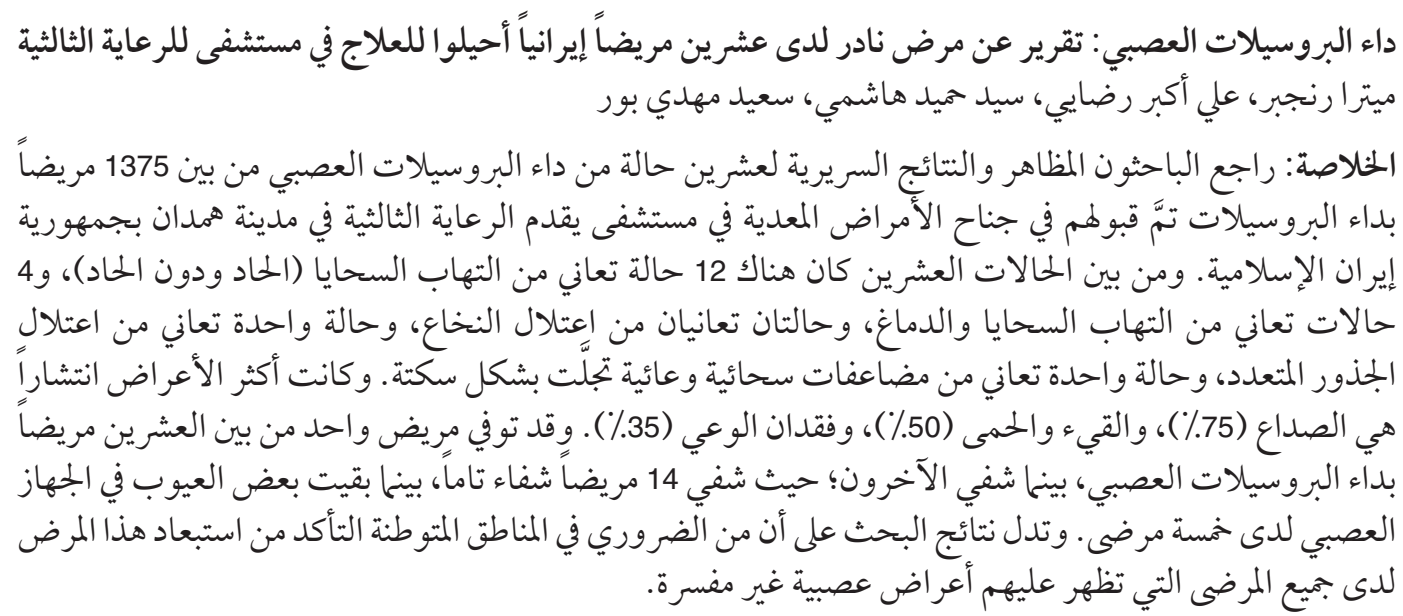

ABSTRACT We reviewed the clinical manifestations and outcome of 20 cases of neurobrocellosis out of 1375 patients with brucellosis admitted to the infectious diseases ward of a tertiary hospital in Hamedan, Islamic Republic of Iran. Of the 20 cases, 12 had meningitis (acute and subacute), 4 had meningoencephalitis, 2 had myelopathy, 1 had polyradiculopathy and 1 had meningovascular complications manifested by stroke. The most prevalent symptoms were headache $(75 \%)$, vomiting and fever $(50 \%)$ and unconsciousness (35\%). Among 20 neurobrucellosis patients, 1 died and the other 19 recovered; 14 fully recovered and 5 patients had residual neurological deficits. In endemic areas, the disease should be ruled out in all patients who develop unexplained neurological symptoms.

Neurobrucellose : enquête sur une maladie rare chez 20 patients iraniens adressés à un hôpital de soins tertiaires

RÉSUMÉ Nous avons étudié les manifestations et l'issue clinique de 20 cas de neurobrocellose parmi 1375 patients atteints de cette maladie admis au service des maladies infectieuses d'un hôpital de soins tertiaires de Hamedan (République islamique d'Iran). Sur ces 20 cas, 12 présentaient une méningite (aiguë et subaiguë), 4 une méningoencéphalite, 2 une myélopathie, 1 une polyradiculopathie et 1 des complications méningovasculaires qui s'étaient manifestées par un accident vasculaire-cérébral. Les symptômes les plus répandus étaient des céphalées (75\%), des vomissements et de la fièvre (50\%) et une perte de connaissance ( $35 \%$ ). Parmi les 20 patients atteints de neurobrucellose, 1 est décédé et les 19 autres se sont rétablis, dont 14 complètement et 5 qui ont conservé des déficits neurologiques résiduels. Dans les régions endémiques, il convient d'écarter cette maladie chez tous les patients présentant des symptômes neurologiques inexpliqués.

${ }^{1}$ Department of Infectious Diseases, Iran University of Medical Sciences, Tehran, Islamic Republic of Iran (Correspondence to M. Ranjbar: ahmaliver@yahoo.com).

${ }^{2}$ Department of Neurology, Sina Hospital, Hamedan, Islamic Republic of Iran.

${ }^{3}$ General Practice, Hamedan, Islamic Republic of Iran.

Received: 26/06/06; accepted: 22/05/07

المجلة الصحية لشرق المتوسط، منظمة الصحة العالمية، المجلد الخامس عشر، العدد (، 9 +. 


\section{Introduction}

Brucellosis is a common zoonosis endemic in many parts of the world [1]. Brucella melitensis remains an important human pathogen in endemic regions, most notably the Mediterranean basin, Arabian peninsula and Indian subcontinent [2,3]. There are frequent reports of brucellosis from countries such as Kuwait, Saudi Arabia and other countries of the Eastern Mediterranean Region, where animals are raised in large numbers $[4,5]$. Brucellosis is an endemic disease in the Islamic Republic of Iran [6]. In 2004 the incidence of brucellosis was 38 per 100000 population. The city of Hamedan in the west of the country has always been considered to have a high incidence of brucellosis (121 per 100000 population). It has many villages with large numbers of goat and sheep farmers and ranchers who ingest unpasteurized dairy products.

Neurobrucellosis is a rare, severe form of systemic infection that has a broad range of clinical syndromes [7-9]. In 4\%-13\% of patients with brucellosis, the central nervous system is involved. $[5,10,11]$. The most frequent presentations of neurobrucellosis include meningitis, meningoencephalitis, polyradiculoneuritis and also cranial nerve palsies.

Considering the high prevalence of brucellosis in the Islamic Republic of Iran, especially in Hamedan province, and the debilitating consequences of neurobrucellosis, we decided to study this aspect of brucellosis to achieve a better understanding of the characteristics and clinical manifestations of this rare, but life-threatening complication.

\section{Methods}

In the review of 1375 hospital records of patients admitted with brucellosis to the in- fectious diseases ward of a tertiary hospital, Hamedan Sina hospital, from 2001 to 2005, 20 patients $(1.45 \%)$ with documented neurobrucellosis were isolated. The diagnostic criteria of neurobrucellosis in these patients were: signs and symptoms of nervous system involvement (headache, nausea, vomiting, lethargy, stupor, coma, hemiplegia, hemiparesia, paraparesia, cerebral nerve palsy, ataxia, behavioural disorder, aphasia, etc.); Brucella agglutination antibody titre $>$ 1:160; pleocytosis [white blood cell (WBC) count in cerebrospinal fluid $\left.(\mathrm{CSF})>10 / \mathrm{m}^{3}\right]$; and clinical improvement with appropriate therapy. Complete records of all the above were required for the diagnosis to be made. A positive blood or CSF culture for Brucella species or a CSF Brucella agglutination antibody titre $>1: 80$ was corroborative, but not required for the diagnosis. Although this was a retrospective study, all the above data were recorded in detail in the archives as standard procedure in our hospital in such cases.

Patient information was gathered, including: clinical presentation; neurological signs; CSF and blood Brucella antibody agglutination titres; cell count, protein level and glucose level in CSF; blood and CSF culture results; dominant clinical manifestations; treatment; and outcome. According to the approach of this hospital, the Brucella antibody agglutination test was performed with the use of kits supplied by the Pasteur Institute of Iran. A serum agglutination titre of $>1: 160$ and a CSF agglutination titre of $>1: 80$ were considered as indicative of the diagnosis. Blood and CSF were cultured on Castaneda biphasic media. Culture bottles were held for up to 6 weeks. Antimicrobial therapy consisted of a combination of 3 to 4 agents used for treatment of brucellosis. The therapeutic regimens included doxycycline, rifampicin and trimethoprimsulfamethoxazole or doxycycline, strepto- 
mycin, trimethoprim-sulfamethoxazole and ceftriaxone.

The data were analysed using the statistical software SPSS, version 9.

\section{Results}

Of 20 patients with neurobrucellosis, 12 $(60 \%)$ were male. As shown in Table 1 , the patients were distributed equally between different age groups, except the 41-60 year age group which contained fewer patients. Among these patients $3(15 \%)$ were single and the rest were married. Most of the patients were farmers $(40 \%)$ or housekeepers (35\%) (Table 1). Eleven patients (55\%) lived in rural areas while the rest were living in cities. Of all patients with neurobrucellosis, $6(30 \%)$ had a previous history of brucellosis.

Table 1 shows the frequency of signs and symptoms of the patients at presentation to the hospital. The most frequent symptoms were headache $(75 \%)$, vomiting and fever $(50 \%)$ and stupor $(35 \%)$. The patients were classified based on the dominant clinical manifestation. Initial clinical manifestations consisted of acute and subacute meningitis in 12 patients, meningoencephalitis in 4, and transverse myelitis, lumbar epidural abscess with radicular nerve compression, polyradiculopathy, and intracerebral haemorrhage each in 1.

All patients underwent lumbar puncture. The mean count of WBC in CSF was $130.2 / \mathrm{mm}^{3}$ (range: $10-580 / \mathrm{mm}^{3}$ ). The mean glucose level was 44.9 (standard deviation $39.37) \mathrm{mg} / \mathrm{dL}$ and the highest protein level was $440 \mathrm{mg} / \mathrm{dL}$. The CSF Brucella antibody agglutination test was performed for 17 patients, and the titre was elevated in 6. Blood culture was performed for 16 patients and was positive for Brucella species in only 1 patient. The blood Brucella antibody agglutination titre was positive in all patients.
The culture results for all 16 patients who had CSF cultures were negative.

Of the 20 patients, 1 died and the other 19 recovered; 14 fully recovered and 5 had a residual neurological deficit. One patient with residual neurological deficit developed psychosis (schizophrenia) despite receiving antimicrobial therapy, 1 had hemiparesis, 1 had depression, 1 had dementia and 1 had permanent hearing loss. The outcome was

Table 1 Characteristics of $\mathbf{2 0}$ neurobrucellosis patients presenting to Hamedan Sina hospital

\begin{tabular}{lrr}
\hline Variable & No. & $\%$ \\
\hline Sex & & \\
Male & 12 & 60 \\
Female & 8 & 40
\end{tabular}

Age group (years)

$\begin{array}{lll}<21 & 6 & 30 \\ 21-40 & 6 & 30 \\ 41-60 & 2 & 10 \\ >60 & 6 & 30\end{array}$

Occupation

$\begin{array}{llr}\text { Farmer } & 8 & 40 \\ \text { Housekeeper } & 7 & 35 \\ \text { Employee } & 2 & 10 \\ \text { Unemployed } & 2 & 10 \\ \text { Student } & 1 & 5\end{array}$

Clinical manifestations

Headache $\quad 15 \quad 75$

Vomiting $10 \quad 50$

Fever $\quad 10 \quad 50$

$\begin{array}{lll}\text { Stupor } & 7 & 35\end{array}$

Blurred vision $\quad 5 \quad 25$

Neck stiffness $\quad 4 \quad 20$

Back pain $\quad 4 \quad 20$

Papilloedema $\quad 3 \quad 15$

Aphasia $3 \quad 15$

Seizure $\quad 3 \quad 15$

Ataxia $\quad 3 \quad 15$

Hemiparesis $\quad 3 \quad 15$

Flaccid paralysis $\quad 2 \quad 10$

Hearing loss $\quad 1 \quad 5$ 
more favourable for those patients who were treated early in their illness.

\section{Discussion}

Neurobrucellosis is a rare manifestation of brucellosis; it is reported that about $4 \%-13 \%$ of patients with brucellosis will develop neurobrucellosis $[5,10,11]$. In our centre, the frequency of neurobrucellosis was lower $(1.45 \%)$ than in previous epidemiological studies. This might be because our centre is a referral centre and we do not have an active screening system to find new cases in the population. Some patients might be referred to neurological clinics and some are not registered if they are not admitted to hospital. In any case, the true frequency of neurobrucellosis is difficult to establish because many patients are not diagnosed and uncomplicated cases are not admitted to hospital. As in any hospital-based retrospective review of medical records, we cannot generalize the findings to the entire population.

In our study the frequency of disease was higher in males, a finding that is compatible with a similar study in the Islamic Republic of Iran [12]. Other investigators have reported a male:female ratio of $2: 1$. In Saudi Arabia the female:male ratio was 2:1 [9]. There was no age preference detected for this disease. Most of the patients were married, lived in rural areas and worked as farmers. This is expected as brucellosis is an occupational disease in shepherds, abattoir workers, veterinarians, dairy-industry professionals and personnel in microbiology laboratories.

Meningoencephalitis can be seen in the acute form and it may also present in chronic form when the peripheral or central nervous system is involved, with epidural granuloma, demyelination of the brain or spinal nerve roots and long tract degeneration.
Meningitis has been reported to be the most frequent presentation, occurring in about $50 \%$ of the cases [13]. In our study, 16 cases $(80 \%)$ had evidence of meningeal infection, as indicated by pleocytosis, elevated protein level in the CSF and usually hypoglycorrhachia. Meningitis and meningoencephalitis were found in 20 $(60 \%)$ and $4(20 \%)$ the cases respectively. Rasoolinejad in a study of 22 Iranian patients with neurobrucellosis found that 10 patients had meningoencephalitis, 7 had meningitis, 3 had polyradiculopathy and 1 presented with spinal epidural abscess and 1 had brain abscess [12]. In a study in Saudi Arabia among 18 patients with neurobrucellosis, $11(61.1 \%)$ patients had meningitis [9]. Koussa et al. found that the most common initial clinical manifestations in 15 cases of neurobrucellosis in France were meningoencephalitis and acute or subacute meningitis in $5(33.3 \%)$ and 4 (26.7\%) patients respectively [14]. In a study in Turkey of 73 patients with brucellosis, 13 patients had neurobrucellosis (17.8\%), 10 had chronic meningitis and 3 had acute meningitis [15]. Bellissma et al. reported that of 6 cases of neurobrucellosis in Italy, 2 had meningoencephalitis, 1 had meningitis with brain abscess and 3 had encephalomyelitis [16].

In our study $2(10 \%)$ patients had myelopathy with pure upper motor neurone syndrome. The peripheral nerve lesions which led to radiculopathy or polyradiculopathy were seen in $1(5 \%)$ patient. In Saudi Arabia, of 18 patients with neurobrucellosis, 1 presented with polyradiculopathy [9]. In another study in France, of 15 patients with neurobrucellosis 2 had polyradiculoneuritis [14]. Goktepe reported a case of neurobrucellosis resembling Guillain-Barré syndrome [17].

Intracerebral and subarachnoid haemorrhage due to a mycotic aneurysm occurred 
in 1 patient. In the study in Saudi Arabia 4 patients had meningovascular complications manifested by stroke or intracerebral haemorrhage [9], a figure that is higher than our results. Tuncer Ertem reported a case of subdural haemorrhage in a 49-year-old female who had neurobrucellosis [18].

Most patients responded favourably to treatment. The most commonly used antibiotics were rifampin, doxycycline and trimethoprim-sulfamethoxazole, in various combinations for at least 3 months. Some patients received streptomycin or ceftriaxone. A regimen consisting of 3 or all 4 of these antimicrobial agents is generally effective for neurobrucellosis, although the addition of steroids has not proven to be consistently beneficial [9]. Corticosteroids seem to protect the tissues from the effects of bacterial toxins and reduce the incidence of long-term complications [19]. The outcome was more favourable for those patients who were treated earlier in the course of the condition. Those who present with prolonged symptoms that last several months may respond more slowly to therapy and have neurological sequelae. Hence it is important to consider the possibility of neurobrucellosis in an endemic region and to treat it aggressively.

In our study $5(25 \%)$ cases had permanent sequelae, 1 of whom $(5 \%)$ died. In McLean et al.'s study of 18 patients, 5 had residual neurological deficits and 4 of them developed permanent hearing loss [9]. In our study 1 patient had permanent hearing loss. In a Nordic study a 20 -year-old Turkish immigrant with spastic paraplegia and hearing loss had neurobrucellosis [20]. Bodur et al. reported 1 case of hearing loss among 13 patients with neurobrucellosis [15].

Neurobrucellosis is an uncommon, serious complication of brucellosis. However, in endemic areas such as Hamedan province, the disease should be ruled out in all patients who develop unexplained neurological symptoms. The clinical signs and symptoms of brucellosis are misleading and many cases are diagnosed as pyrexia of unknown origin $[21,22]$. Physicians and health workers in endemic areas need to be alert to the possibility of the disease and have access to suitable laboratory facilities for diagnosis.

\section{References}

1. Mandell GL, Bennett JE, Dolin R, eds. Mandell, Douglas and Bennet's Principles and practice of infectious diseases, 6th ed. Philadelphia, Churchill Livingstone, 2005.

2. Corbel MJ. Brucellosis: an overview. Emerging infectious diseases, 1997, 3:213-21.

3. Boschiroli ML, Foulongne V, O'Callaghan D. Brucellosis: a worldwide zoonosis. Current opinion in microbiology, 2001, 4:58-64.

4. Bashir R et al. Nervous system brucellosis: diagnosis and treatment. Neurology, 1985, 35:1576-81.
5. Lulu AR et al. Human brucellosis in Kuwait: a prospective study of 400 cases. Quarterly journal of medicine, 1988, 66(249):39-54.

6. Azizi F. The epidemiology of common disease in Iran. Tehran, Islamic Republic of Iran, Endocrinology Research Centre, Shahid Beheshti Medical University, 1993.

7. Shakir RA et al. Clinical categories of neurobrucellosis. A report on 19 cases. Brain, 1987, 110:213-23.

8. Habeeb YK et al. Paediatric neurobrucellosis: case report and literature review. Journal of infection, 1998, 37:59-62. 
9. McLean DR, Russell N, Khan MY. Neurobrucellosis clinical and therapeutic features. Clinical infectious diseases, 1992, 15(4):582-90.

10. Kochar DK et al. Clinical profile of neurobrucellosis-a report on 12 cases from Bikaner (north-west India). Journal of the Association of Physicians of India, 2000, 48(4):376-80.

11. Pascual $\mathrm{J}$ et al. Localized CNS brucellosis: report of 7 cases. Acta neurologica scandinavica, 1988, 78:282-9.

12 Rasoolinejad M. [Neurobrucellosis: clinical and laboratory findings in 22 patients.] Journal of Tehran Faculty of Medicine, 1378, 4(57):92-87 [in Farsi].

13 Al Deeb SM et al. Neurobrucellosis: clinical characteristics, diagnosis, and outcome. Neurology, 1989, 39(4):498-501.

14. Koussa $S$ et al. Neurobrucellose: etudes clinique et therapeutique de 15 patients [Neurobrucellosis: clinical features and therapeutic responses in 15 patients]. Revue neurologique, 2003, 159(12):1148-55.

15. Bodur $\mathrm{H}$ et al. Neurobrucellosis in an endemic area of brucellosis. Scandinavian journal of infectious diseases, 2003, 35(2):94-7.

16. Bellissima P, Turturici MA. Neurobrucellosi: aspetti clinici e terapeutici
[Neurobrucellosis:clinical and therapeutic features]. Le infezioni in medicina, 1998, 6(1):25-30.

17. Goktepe AS et al. Neurobrucellosis and a demonstration of its involvement in spinal roots via magnetic resonance imaging. Spinal cord, 2003, 41(10):574-6.

18. Tuncer Ertem G, Tulek N, Yetkin MA. Olgu raporu: subdural kanama ile seyreden norobrusellozis [Case report: subdural hemorrhage in neurobrucellosis]. Mikrobiyoloji bulteni, 2004, 38(3):253-6.

19. Tunkel AR, Wispelwey B, Scheld WM. Bacterial meningitis: recent advances in pathophysiology and treatment. Annals of internal medicine, 1990, 112:610-23.

20. Bucher A, Gaustad P, Pape E. Chronic neurobrucellosis due to Brucella melitensis. Scandinavian journal of infectious diseases, 1990, 22(2):223-6.

21. Ergönül $O$ et al. Revised definition of "fever of unknown origin": limitations and opportunities. Journal of infection, 2005, 50:1-5.

22. Al-Eissa $Y$ et al. Childhood brucellosis: a deceptive infectious disease. Scandinavian journal of infectious diseases, 1991, 23:129-33. 